\title{
Telemedicine in Pediatric Headache: A Review and Practical Implementation
}

\author{
Scott Rosenthal ${ }^{1}$ (D) Marcy Yonker ${ }^{2}$ \\ Accepted: 24 March 2021 / Published online: 12 April 2021 \\ (C) The Author(s), under exclusive licence to Springer Science+Business Media, LLC, part of Springer Nature 2021
}

\begin{abstract}
Purpose of Review The purpose of this review was to summarize the current literature about telemedicine in pediatric headache and to provide practical guidance for its implementation.

Recent Findings There are few studies dedicated to telemedicine in pediatric headache, and existing studies are small. Patients and families report high levels of satisfaction with telemedicine, and most are willing to continue telemedicine visits in the future. Telemedicine demonstrated similar reductions in headache frequency, severity, and duration as patients treated in-person. Remotely delivered psychologic interventions have some utility in reducing headache severity acutely. Families feel telemedicine reduces geographic and financial barriers to care.

Summary Telemedicine in pediatric headache is a growing field. While there is limited research available, it appears safe, efficacious, and feasible. Headache-related outcomes, including frequency, severity, and duration, were similar amongst telemedicine and in-person visits. Future studies should include larger sample sizes and detailed analysis of adverse outcomes.
\end{abstract}

Keywords Telemedicine $\cdot$ Telehealth $\cdot$ Teleneurology $\cdot$ Pediatric headache $\cdot$ Pediatric migraine $\cdot$ Headache

\section{Introduction}

Migraines represent a common cause of disability in the US affecting approximately 28 million individuals [1]. Approximately 1 out 10 of these individuals is adolescent, resulting in approximately 1 million missed school days per year [2]. While headache clearly causes significant disability, only a small percentage of these individuals seek care in specialized clinics or hospital settings. It is estimated that only 5 percent of those with migraines receive the comprehensive medical care they deserve $[3,4 \cdot \bullet]$. Despite this, headaches still represent the second most common reason for referral to pediatric neurology [1]. Unfortunately, there are a vast shortage

This article is part of the Topical Collection on Headache

Scott Rosenthal

Scott.rosenthal@childrenscolorado.org

1 Children's Hospital Colorado, University of Colorado School of Medicine, 13123 E 16th Ave., Aurora, CO 80045, USA

2 Pediatric Headache Program Children's Hospital Colorado, University of Colorado School of Medicine, 13123 E 16th Ave., Aurora, CO 80045, USA of pediatric neurologists and an even greater shortage of pediatric neurologists specialized in headache [5]. This is exacerbated by the propensity of pediatric neurologists to cluster in metropolitan communities and near academic centers, thus placing those living in rural areas at a great disadvantage [6]. Telemedicine represents a mechanism to bypass regional disparities, the relative scarcity of pediatric neurologists, and numerous additional barriers to neurologic care [3].

Telemedicine is defined as the remote delivery of direct patient care via a telecommunications system which typically includes both audio and visual communications [7]. This can occur via synchronous telemedicine occurring in real time or via asynchronous telemedicine, which occurs in a store and forward manner. Synchronous telemedicine may or may not involve a telepresenter, an individual present at the patient's location who is trained to aid in remote examination [7]. Additionally, telemedicine can either utilize a "hub and spoke model" or merely access patients directly in their home. In a "hub and spoke model", a centralized "hub" provides specialized telehealth to patients at a remote "spoke" medical care facility $[8 \cdot]$.

The rapid spread and development of information and telecommunication technologies over recent years have offered numerous opportunities for the expansions of telemedicine 
[3]. This process has been catapulted into widespread use in the setting of the ongoing novel coronavirus (COVID19) pandemic [9•]. This has not only resulted in increased access to care but has also ensured a safe way to maintain follow-up during an ongoing crisis [10]. Telemedicine also disassembles numerous barriers experienced by families seeking neurologic expertise. Many families live in rural areas and must coordinate traveling vast distances to receive care. This often is not only limited to the physical act of traversing a long distance but also coordinating childcare for siblings, taking time off from work/school, and the financial costs of travel itself [3, $6,11]$. Even after these barriers are overcame, families often face difficulties navigating unfamiliar facilities and parking [6]. All of this is often further exacerbated by physical disabilities, or behavioral challenges often present in the pediatric neurology population [7]. Telemedicine offers an easy alternative in the face of such challenges.

\section{Prior Successes of Telemedicine}

While the utilization of telemedicine in pediatric headaches is still evolving, Teleneurology itself has been well established since 1999 [12]. Teleneurology, the remote delivery of neurology, formed its roots in the field of adult stroke management. The high-risk nature of acute stroke and the need to expand access to life-saving and disability preventing intravenous (IV) tissue plasminogen activator (TPA) jettisoned the widespread implementation of Teleneurology [7, 13, 14].

Several studies have found equivalent outcomes amongst telemedicine-based evaluation and administration of IV TPA when compared to those treated in-person [15-17]. Likewise, studies have found similar rates for remotely delivered TPA complications $[15,17]$. Many studies have also demonstrated excellent accuracy of remote stroke examinations with strongly correlated telemedicine and in-person NIH Stroke Scales [18-20]. One study even found the diagnostic accuracy of strokes in telemedicine to be 98 percent [21]. Outside of strokes, interrater reliability of general neurology telemedicine examinations has also been shown to be similar to those performed in-person [22].

In addition to stroke, Teleneurology has been utilized in a wide array of neurologic conditions including epilepsy [23, 24], multiple sclerosis [25], neurodegenerative disorders [26, 27], and adult headache $[11,28 \bullet, 29,30]$. Amongst 135 neurologists surveyed during the COVID19 pandemic, headache was viewed as one of the specialties most suited for telehealth follow-up due to its emphasis on clinical history [10]. This is well supported by recent telemedicine studies in adult headache [28• 29].

A study of 30 individuals with migraine, randomized to inperson visits or telemedicine, found equivalent reductions in headache severity, frequency, and migraine disability assessment scores (MIDAS) at 1 year. Furthermore, the individuals in the telemedicine group rated their visits as more convenient [28 [ $^{\circ}$. A similar study of 102 individuals with medication overuse headache found no difference in headache frequency nor severity amongst those treated via telemedicine at 3-and 12-month follow-up [29]. The results of both of these studies are further supported by a large study of 402 new headache consultations randomized to telemedicine or inperson visits. This study found no difference in headache disability or pain scores at 3 and 12 months. At 12-month followup, these subjects also demonstrated equivalent satisfaction rates to those receiving in-person visits [30]. Furthermore, at 1 year there was no difference in the frequency of secondary headaches, lumbar puncture results, nor hospital stays for either group. This study estimated that the number of telemedicine visits needed to miss 1 secondary headache was approximately 20,200 [30]. This suggests not only is telemedicine efficacious in adult headache but it is also exceedingly safe.

Much like adult telemedicine, pediatric telemedicine has also been proven safe and efficacious. The American Academy of Pediatrics has supported telemedicine as a mechanism to fill provider gaps since 2015 [8•], and a few studies have demonstrated equivalent or better outcomes with telemedicine [31, 32]. A study of 223 children with ADHD, age 5 to 13 years, found equivalent outcomes and patient/parent satisfaction when compared to in-person visits [32]. In another large study, 400 children with asthma, age 3-10 years, were randomized to school-based telemedicine or enhanced traditional care which found that the telemedicine group had a lower frequency of symptomatic days, were less likely to be hospitalized, and had fewer emergency department visits [31].

\section{Telemedicine in Pediatric Headache}

\section{Feasibility and Efficacy}

Telemedicine has become an increasingly important component of both adult and pediatric medical cares. This has been expedited by the current COVID19 pandemic and the relative scarcity of specialist providers. Pediatric neurologists are not exempt from this, especially those managing pediatric headache. Unfortunately, to date, only a few studies have examined the use of telemedicine in pediatric headache; however, all have shown excellent promise $[4 \bullet \bullet, 8 \bullet, 26,33 \bullet, 34 \bullet$.

A prospective study of 51 established pediatric migraine patients, age 5-18 years, followed their outcomes and patient satisfaction with telemedicine over a 70 -day period [33•]. This study found greater than 90 percent of caregivers reported a high level of satisfaction with telemedicine and understanding of the providers' instructions. 85-90 percent of caregivers reported that they felt telemedicine was as efficacious as inperson visits and would like to continue telemedicine after 
resolution of the COVID19 pandemic. Objectively, 90 percent of children were compliant with medications and 80 percent were compliant with lifestyle modifications. Unfortunately, this study was limited by its small size, exclusion of new patients, and exclusion of non-migraine headache diagnoses [33•]. A similar study of 7 patients, age 7-17 years, evaluated new headache patients via an initial in-person visit and, subsequently, followed up monthly via a telemedicine hub and spoke model for 3 months [8•]. This study found high patient and parent satisfaction with both averaging $4.8 / 5$ on a Likert scale ( $5 / 5$ being "very satisfied"). This study also found a no show rate of 8 percent for telemedicine, similar to 13 percent for in-person clinics. Headache symptom improvement was equivalent as well. This study was limited by its small size and exclusion of comorbid psychiatric diagnoses [8•], and both studies were limited by the lack of a randomized control group $[8 \cdot, 33 \cdot]$. However, both studies did show similar satisfaction rates and outcomes as those seen in adult headache telemedicine literature $[28 \bullet, 29,35]$.

\section{Safety}

Objective safety data on telemedicine in pediatric headache is quite limited. Few studies report rates of adverse outcomes. In the aforementioned study of 51 headache patients, the majority of adverse events were related to insufficient response to current medications and only 2 patients required treatment in an emergency department. Furthermore, only 10 percent of all unplanned follow-up calls were requests for medication changes due to side effects [33•]. A more comprehensive evaluation of the safety in pediatric telemedicine found that only 0.7 percent of over 2000 telemedicine patients were admitted to the hospital within 7 days of the visit. Within 8-30 days of the visit, 0.8 percent of patients were admitted and no deaths were seen throughout the entire study. While this study analyzed all pediatric neurology patients seen via telemedicine, at least 594 of them were diagnosed with headache or migraines [34•]. This study also found that there was no difference in the rate of subsequently performed in-person examinations nor hospitalizations when comparing new and established telemedicine patients [34॰]. This suggests telemedicine is indeed safe for new pediatric headache patients. This is in agreement with an adult study of 402 new headache patients randomized to telemedicine or in-person evaluation. Both groups had equivalent headache outcomes, neurodiagnostic findings, and rates of secondary headaches [29]. The evaluation of new patients via telemedicine is often viewed with anxiety due to the difficulty performing a comprehensive neurologic examination and ruling out secondary processes [10, 26, 33•]; however, both of these studies suggest that telemedicine is safe and adequate for this process [29, 34•]. Additionally, it is felt that careful screening for red flag symptoms and referral to local specialists when indicated are sufficient to overcome this barrier $[6,9 \bullet, 30,33 \bullet]$. Finally, it is worthwhile to reiterate that secondary causes of headache are rare and the number of telemedicine visits necessary to miss one secondary headache is estimated at 20,200 [30].

\section{Multimodal Approaches to Headache}

Outside of the classic neurology provider and patient/family paradigm, telemedicine has several additional uses in the remote management of headache. Telemedicine can be utilized to teach non-pharmaceutical interventions for headaches $[1$, $\left.36,37{ }^{\circ}\right]$ and can be utilized to further encourage appropriate headache hygiene strategies $[33 \cdot 36]$.

A study of 34 teenagers with migraines randomized patients to either a telephone-based behavioral migraine treatment education group or a standard triptan treatment group [1]. The behavioral strategy group was able to demonstrate good mastery of the techniques learned over the telephone. 80 percent of patients endorsed high satisfaction and a preference for telemedicine visits over in-person visits. Additionally, 90 percent of teenagers in the behavioral treatment group felt like they had a good relationship with their tele-counselor. This group averaged a reduced headache frequency of greater than 50 percent reduction at 3 months and nearly 80 percent reduction at 8 months. While this study was small in nature, the behavioral treatment group demonstrated similar reductions in migraine frequency, disability, severity, and improvements in quality of life to those treated purely with triptans. This improvement in migraine severity is supported by a recent Cochrane review of remotely delivered psychological therapies in pediatric chronic pain [37•]. This review identified and pooled data from 5 headache studies [1, 38-41] and 2 studies [42, 43] comprised of headache pain and mixed chronic pain syndromes. For the headache-specific studies, a statistically and clinically significant reduction in headache severity was observed immediately after remotely delivered psychologic therapies. Furthermore, this review found that the number needed to treat to achieve this reduction was merely 2.88 . Amongst the 2 mixed pain studies, headache intensity was also significantly reduced post-treatment. Unfortunately, this effect was not sustained in subsequent follow-up. Additionally, no significant effect on headache related disability, depression, or anxiety was found [37•]. While not directly evaluated, the results of these studies suggest that there may be a place for a multi-modal telemedicine approach in the treatment of pediatric headaches.

In addition to the ability to remotely teach and deliver nonpharmacologic headache interventions, telemedicine can likely be utilized to encourage lifestyle modifications as well. With ever increasing access to smartphones, more and more medical conditions are benefitting from the development of illness related smartphone applications. Headaches are no exception to this [44]. A recent study of pediatric migraine 
successfully tracked lifestyle and medication adherence via an electronic monitoring system and smartphone-based self-report [36]. Although this study merely tracked adherence, it logically follows that these could easily be used to encourage and reinforce headache lifestyle modifications.

\section{Additional Benefits}

Telemedicine yields numerous additional benefits beyond those directly related to the management of headaches. The most immediate and obvious of those are related to access and travel. Patients' families often report that telemedicine is more convenient $[4 \bullet \bullet, 7,8 \bullet$, causes less disruption of their daily routine $[4 \bullet \bullet, 26]$, and reduces the need to navigate large geographic obstacles with frequently challenging weather [26]. Not having to travel also abates the need for coordinating care for additional children [26, 33•], taking time off from work, school, or extra-curricular activities [8•]. Furthermore, by not traveling, families evade all the associated financial burdens that stem from traveling itself, such as lodging, parking, meals, income missed due to time away from work, and numerous additional costs $[8 \bullet, 26,33 \bullet]$. While this seems intuitively true, families also report that they felt telemedicine saved them money [4••, 26, 33•]. In one study of pediatric migraine patients seen via telemedicine, families reported an average savings of $\$ 486$ per visit [4••]. A similar study of pediatric concussions, a condition in which headache is exceedingly common [45], estimated that families saved nearly $\$ 41,000$ across 57 telemedicine consults and subsequent phone follow-ups [26]. Additionally, travel is a common trigger for pediatric migraines and avoidance of this reduces unnecessary patient discomfort and familial stress $[4 \bullet \bullet, 33 \bullet]$. Telemedicine also reduces waiting times to see specialists, which can further decrease undue discomfort and stress [4••, 7, 11].

In addition to the benefits telemedicine provides to patients and families, telemedicine also benefits medical providers. Telemedicine has been shown to have lower

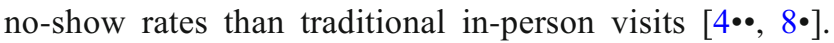
Telemedicine clinics are also more likely to run on time and typically have fewer late arrivals [4••]. In a study of pediatric headache via a hub and spoke model, the local staff felt the telemedicine improved their understanding of complex headache management [8•]. Finally, telemedicine provides a mechanism for providers to maintain a therapeutic relationship even at a distance [7].

\section{Common Concerns about Telemedicine and Headache}

The most common concern in telemedicine is the limitations it imposes on the comprehensive neurologic examination $[6,8 \bullet, 26,33 \bullet]$. While certain maneuvers can easily be performed over telemedicine, evaluation of tone, deep tendon reflexes, and fundoscopy are all nearly impossible without the presence of an experienced telepresenter [6, $8 \bullet, 27]$. In cases when such an evaluation is felt necessary, referral to local providers, such as ophthalmology or a primary care provider, can circumvent this problem. When this is not feasible, planned in-person follow-up is a reasonable alternative [6]. Outside of the concerns surrounding remote examinations, many providers have concerns about the technologic side of telemedicine $[1,4 \bullet \bullet$, $8 \bullet, 34 \bullet]$. This seems unfounded as rates of audio-visual malfunctions were rare [8•, 34•]. In fact, out of over 2000 pediatric neurology telemedicine appointments, only 1 percent experienced a technical challenge necessitating transition to a telephone call [34•]. Additionally, this study found no increase in the rates of telemedicine failure following rapid adoption and expansion for the COVID19 pandemic [34•].

\section{Practical Implementation of Telemedicine in Headache}

The approach to a headache telemedicine visit can largely be performed in a similar manner to typical in-person visits [8•]. Prior to the visit, patients and families can complete relevant screening forms which can subsequently be uploaded into the electronic medical record (EMR). Likewise, relevant imaging discs and tests can be mailed or digitally transferred to the EMR before the visit. To reduce the frequency of audio-video malfunctions, patients should be provided with either stepwise instruction for telemedicine access or an equipment check by a trained information and technology specialist. For individuals without access to internet or smart devices, travel to a local hub site or a purely telephone-based interview followed by in-person evaluation may be necessary. Vitals are not easily obtained unless the patient is at a spoke site or if in-home monitoring devices are present; however, these are often not necessary for clinical decision-making in headache. If such information is needed, patients can be referred to local providers for further evaluation.

After establishment of audio-visual connection via one's preferred HIPPA compliant platform, the history of present illness, review of systems, and review of past medical history can be obtained in the standard fashion. As always, one should take care to screen for red flag symptoms that may indicate the presence of a secondary cause of headache $[6,9 \bullet, 30,33 \bullet]$. The physical examination requires slight adjustment, but the core components remain the same. The patient should be centered in front of a well-lit camera. Mental status and speech are evaluated in the usual manner. After positioning the patient's 
face centrally in front of the camera, much of the cranial nerve evaluation remains unchanged; however, extraocular movements must be tested by instructing the patient to look in various directions and the pupillary exam is limited to consideration of symmetry. Funduscopic evaluation is untestable and, if indicated, should be evaluated by local providers or in dedicated in-person followup. The motor examination is purely observational; however, the patient can demonstrate anti-gravity maneuvers, and likewise, pronator drift can be tested. Sensation can be evaluated by patient report and self-stimulation; unfortunately, more extensive modalities are typically unavailable. Reflexes and tone cannot be assessed unless a telepresenter is present. Coordination can be tested by observation of rapid alternating movement, and screening for dysmetria can be performed in a modified finger-nosefinger test. This is performed by picking a fixed location as a substitute for the examiner's finger and then performing the maneuver in the usual fashion. Finally, gait and station are assessed in the standard fashion, provided there is sufficient distance from the camera and room to ambulate. Of note, extra caution should be taken prior to initiation of this testing as one may not be present to catch particularly unsteady patients. During the examination itself, it is often necessary to provide feedback about patient and camera orientation to optimize visualization, and repeat instructions are often helpful. If any examination components are insufficient, ambiguous, or abnormal, patients can undergo repeat examination by local providers or via dedicated in-person follow-up as indicated $[8 \bullet]$.

\section{Conclusion}

Telemedicine offers a mechanism to increase patient access to specialty care by reducing financial, geographic, and logistical barriers. The field of neurology had early success with the implementation telemedicine in stroke over 20 years ago; however, widespread adoption has been slow and even more so amongst pediatric neurology. This slow adoption was compounded by the shortage of pediatric headache specialists; however, the COVID19 pandemic has led to the rapid implementation and expansion of telemedicine. While the current literature on telemedicine in pediatric headache is quite limited, current evidence suggests that telemedicine is effective in the treatment of headaches, is safe for new and established patients, and is well perceived by patients and their families. Furthermore, there is evidence that telemedicine can be utilized to distribute non-pharmacologic interventions and patients may benefit from a multi-modal approach. Telemedicine visits are easily completed in a similar manner to typical in-person visits with only slight adjustments necessary during the physical examination.

\section{Declarations}

Human and Animal Rights This article does not contain any studies with human or animal subjects performed by any of the authors.

Conflict of Interest The authors declare that they have no conflict of interest.

\section{References}

Papers of particular interest, published recently, have been highlighted as:

- Of importance

- Of major importance

1. Cottrell C, Drew J, Gibson J, Holroyd K, O’Donnell F. Feasibility assessment of telephone-administered behavioral treatment for adolescent migraine. Headache. 2007;47:1293-302. https://doi.org/ 10.1111/j.1526-4610.2007.00804.x.

2. Powers S, Patton SR, Hommel KA, Hershey AD. Quality of life in childhood migraines: clinical impact and comparison to other chronic illnesses. Pediatrics. 2003;112:e1-5. https://doi.org/10. 1542/peds.112.1.e1.

3. Akiyama H, Hasegawa Y. A trial case of medical treatment for primary headache using telemedicine. Medicine (Baltimore). 2018;97:e9891. https://doi.org/10.1097/MD.0000000000009891.

4.• Qubty W, Patniyot I, Gelfand A. Telemedicine in a pediatric headache clinic: a prospective survey. Neurology. 2018;19:e1702-5. https://doi.org/10.1212/WNL.0000000000005482. Largest prospective study of telemedicine in pediatric headache to date. Found telemedicine to be convenient, cost-effective and have high levels of satisfaction.

5. Dall TM, Storm MV, Chakrabarti R, et al. Supply and demand analysis of the current and future US neurology workforce. Neurology. 2013;81:470-8. https://doi.org/10.1212/WNL. 0000000000000509.

6. Velasquez SE, Chaves-Carballo E, Nelson EL. Pediatric teleneurology: a model of epilepsy care for rural populations. Pediatr Neurol. 2016;64:32-7. https://doi.org/10.1016/j. pediatrneurol.2016.08.001.

7. García-Pérez A. Telemedicina en neuropediatría [Telemedicine in pediatric neurology]. Rev Neurol. 2020;71:191-6. Spanish. https:// doi.org/10.33588/rn.7105.2020304.

8. Vierhile A, Tuttle J, Adams H, tenHoopen C, Baylor E. Feasibility of providing pediatric neurology telemedicine care to youth with headache. J Pediatr Health Care. 2018;32:500-6. https://doi.org/10. 1016/j.pedhc.2018.02.004. Small study of pediatric headache managed via hub and spoke model. Patients followed over 3 months had equivalent headache outcomes to traditional visits and high parent/patient satisfaction.

9. Duncan C, Macleod AD. Video consultations in ordinary and extraordinary times. Pract Neurol. 2020;20:396-403. https://doi.org/ 10.1136/practneurol-2020-002579. Small study of telemedicine in pediatric headache. Demonstrated high patient and caregiver satisfaction. Demonstrated feasibility with minimal technologic complications. 
10. Kristoffersen ES, Sandset EC, Winsvold BS, Faiz KW, Storstein AM. Experiences of telemedicine in neurological out-patient clinics during the COVID-19 pandemic. Ann Clin Transl Neurol. 2021;8: 440-7. https://doi.org/10.1002/acn3.51293.

11. Muller KI, Alstadhaug KB, Bekkelund SI. Telemedicine in the management of non-acute headaches: a prospective, open-labelled non-inferiority, randomised clinical trial. Cephalalgia. 2017;37: 855-63. https://doi.org/10.1177/0333102416654885.

12. Levine SR, Gorman M. "Telestroke": the application of telemedicine for stroke. Stroke. 1999;30:464-9. https://doi.org/10.1161/01. str.30.2.464.

13. Amorim E, Shih MM, Koehler SA, Massaro LL, Zaidi SF, Jumaa $\mathrm{MA}$, et al. Impact of telemedicine implementation in thrombolytic use for acute ischemic stroke: the University of Pittsburgh Medical Center telestroke network experience. J Stroke Cerebrovasc Dis. 2013;22:527-31. https://doi.org/10.1016/j.jstrokecerebrovasdis. 2013.02.004.

14. Müller-Barna P, Hubert GJ, Boy S, Bogdahn U, Wiedmann S, Heuschmann PU, et al. TeleStroke units serving as a model of care in rural areas: 10-year experience of the TeleMedical Project for integrative Stroke Care. Stroke. 2014;45:2739-44. https://doi.org/ 10.1161/STROKEAHA.114.006141.

15. Zaidi SF, Jumma MA, Urra XN, Hammer M, Massaro L, Reddy V, et al. Telestroke-guided intravenous tissue-type plasminogen activator treatment achieves a similar clinical outcome as thrombolysis at a comprehensive stroke center. Stroke. 2011;42:3291-3. https:// doi.org/10.1161/STROKEAHA.111.625046.

16. Johansson T, Wild C. Telerehabilitation in stroke care-a systematic review. J Telemed Telecare. 2011;17:1-6. https://doi.org/10. 1258/jtt.2010.100105.

17. Kepplinger J, Barlinn K, Deckert S, Scheibe M, Bodechtel U, Schmitt J. Safety and efficacy of thrombolysis in telestroke: a systematic review and meta-analysis. Neurology. 2016;87:1344-51. https://doi.org/10.1212/WNL.0000000000003148.

18. Handschu R, Scibor M, Willaczek B, STENO Project, et al. Telemedicine in acute stroke: remote video-examination compared to simple telephone consultation. J Neurol. 2008;255:1792-7. https://doi.org/10.1007/s00415-008-0066-9.

19. Meyer BC, Lyden PD, Al-Khoury L, et al. Prospective reliability of the STRokE DOC wireless/site independent telemedicine system. Neurology. 2005;64:1058-60. https://doi.org/10.1212/01.wnl. 0000209203.87339.21.

20. Rubin MJ. Teleneurology report card: proof of concept. Pract Neurol. 2011:32-4 Retrieved from http://practicalneurology.com/ 2011/06/viewpointsteleneurology-report-card-proof-of-concept/.

21. Meyer BC, Raman R, Hemmen T, Obler R, Zivin J, et al. Efficacy of site-independent telemedicine in the STRokE DOC trial: a randomised, blinded, prospective study. Lancet Neurol. 2008;7: 787-95. https://doi.org/10.1016/S1474-4422(08)70171-6.

22. Craig JJ, McConville JP, Patterson VH, Wootton R. Neurological examination is possible using telemedicine. J Telemed Telecare. 1999;5:177-81. https://doi.org/10.1258/1357633991933594.

23. Haddad N, Grant I, Eswaran H. Telemedicine for patients with epilepsy: a pilot experience. Epilepsy Behav. 2015;44:1-4. https://doi.org/10.1016/j.yebeh.2014.11.033.

24. Ahmed SN, Wiebe S, Mann C, Ohinmaa A. Telemedicine and epilepsy care - a Canada wide survey. Can J Neurol Sci. 2010;37: 814-8. https://doi.org/10.1017/s0317167100051490.

25. Kane RL, Bever CT, Ehrmantraut M, Forte A, Culpepper WJ, Wallin MT. Teleneurology in patients with multiple sclerosis: EDSS ratings derived remotely and from hands-on examination. $\mathrm{J}$ Telemed Telecare. 2008;14:190-4. https://doi.org/10.1258/jtt. 2008.070904.

26. Ellis MJ, Boles S, Derksen V, Dawyduk B, Amadu A, Stelmack K, et al. Evaluation of a pilot paediatric concussion telemedicine programme for northern communities in Manitoba. Int J Circumpolar
Health. 2019;78:1573163. https://doi.org/10.1080/22423982.2019. 1573163.

27. Wechsler LR. Advantages and limitations of teleneurology. JAMA Neurol. 2015;72:349-54. https://doi.org/10.1001/jamaneurol.2014. 3844.

28. Friedman DI, Rajan B, Seidmann A. A randomized trial of telemedicine for migraine management. Cephalalgia Int $\mathrm{J}$ Headache. 2019;39:1577-85. https://doi.org/10.1177/0333102419868250. Randomized adult migraine patients to telemedicine or inperson visits. Found equivalent MIDAS scores, number of headache days, and average severity at 1 year follow-up.

29. Bekkelund SI, Müller KI. Video consultations in medication overuse headache. A randomized controlled trial. Brain Behav. 2019;9: e01344. https://doi.org/10.1002/brb3.1344.

30. Müller KI, Alstadhaug KB, Bekkelund SI. A randomized trial of telemedicine efficacy and safety for nonacute headaches. Neurology. 2017;89:153-62. https://doi.org/10.1212/WNL. 0000000000004085.

31. Halterman JS, Fagnano M, Tajon RS, Tremblay P, Wang H, Butz $\mathrm{A}$, et al. Effect of the school-based telemedicine enhanced asthma management (SBTeam) program on asthma morbidity: a randomized clinical trial. JAMA Pediatr. 2018;172:e174938. https://doi. org/10.1001/jamapediatrics.2017.4938.

32. Ellington E. Telepsychiatry by APRNs: an answer to the shortage of pediatric providers? Issues Ment Health Nurs. 2013;34:719-21. https://doi.org/10.3109/01612840.2013.784386.

33. Sharawat IK, Panda PK. Caregiver satisfaction and effectiveness of teleconsultation in children and adolescents with migraine during the ongoing COVID-19 pandemic. J Child Neurol. 2021;36:296303. https://doi.org/10.1177/0883073820968653. Followed 51 pediatric headache patients over 146 telemedicine visits. Demonstrated high caregiver satisfaction with telemedicine, feasibility and minimal adverse outcomes.

34. Joshi CN, Yang ML, Eschbach K, Tong S, Jacobson MP, Stillman C, et al. Quality and safety analysis of 2999 telemedicine encounters during the COVID-19 pandemic. Neurol Clin Pract. 2020;10: 1212. https://doi.org/10.1212/CPJ.0000000000001025. Analyzed over 2000 pediatric neurology telemedicine visits during the COVID19 pandemic. Found no difference in telemedicine efficacy and safety compared to telemedicine before the pandemic. Demonstrated low hospitalization rates and no deaths at 30 days post- telemedicine visits.

35. Müller KI, Alstadhaug KB, Bekkelund SI. Headache patients' satisfaction with telemedicine: a 12-month follow-up randomized noninferiority trial. Eur J Neurol. 2017;24:807-15. https://doi.org/10. 1111/ene.13294.

36. Kroon Van Diest AM, Ramsey R, Aylward B, Kroner JW, Sullivan SM, Nause K, et al. Adherence to biobehavioral recommendations in pediatric migraine as measured by electronic monitoring: the Adherence in Migraine (AIM) Study. Headache. 2016;56:113746. https://doi.org/10.1111/head.12836.

37. Fisher E, Law E, Palermo TM, Eccleston C. Psychological therapies (remotely delivered) for the management of chronic and recurrent pain in children and adolescents. Cochrane Database Syst Rev. 2015;3:CD011118. https://doi.org/10.1002/14651858.CD011118. pub2. Cochrane review of remotely delivered psychologic behavioral interventions in 5 headache pain studies and 2 mixed headache pain studies. Found remotely delivered interventions were effective in reducing pain severity.

38. Connelly M, Rapoff MA, Thompson N, Connelly W. Headstrong: a pilot study of a CD-ROM intervention for recurrent pediatric headache. J Pediatr Psychol. 2006;31:737-47. https://doi.org/10.1093/ jpepsy/jsj003.

39. McGrath PJ, Humphreys P, Keene D, Goodman JT, Lascelles MA, Cunningham J, et al. The efficacy and efficiency of a self- 
administered treatment for adolescent migraine. Pain. 1992;49: 321-4. https://doi.org/10.1016/0304-3959(92)90238-7.

40. Rapoff MA, Connelly M, Bickel JL, Powers SW, Hershey AD, Allen JR, et al. Headstrong intervention for pediatric migraine headache: a randomized clinical trial. J Headache Pain. 2014;15:1-10. https://doi.org/10.1186/1129-2377-15-12.

41. Trautmann E, Kroner-Herwig B. A randomized controlled trial of internet-based self-help training for recurrent headache in childhood and adolescence. Behav Res Ther. 2010;48:28-37. https://doi.org/ 10.1016/j.brat.2009.09.004

42. Hicks CL, von Baeyer CL, McGrath PJ. Online psychological treatment for pediatric recurrent pain: a randomized evaluation. J Pediatr Psychol. 2006;31:724-36. https://doi.org/10.1093/jpepsy/jsj065.

43. Palermo TM, Wilson AC, Peters M, Lewandowski A, Somhegyi H. Randomized controlled trial of an internet delivered family cognitive behavioral therapy intervention for children and adolescents with chronic pain. Pain. 2009;146:205-13. https://doi.org/10. 1016/j.pain.2009.07.034.

44. Minen MT, Stieglitz EJ, Sciortino R, Torous J. Privacy issues in smartphone applications: an analysis of headache/migraine applications. Headache. 2018;58:1014-27. https://doi.org/10.1111/head. 13341.

45. Blume HK. Headaches after concussion in pediatrics: a review. Curr Pain Headache Rep. 2015;19:42. https://doi.org/10.1007/ s11916-015-0516-x.

Publisher's Note Springer Nature remains neutral with regard to jurisdictional claims in published maps and institutional affiliations. 